\title{
A Fault Detection Filtering for Networked Control Systems Based on Balanced Reduced-Order
}

\author{
Da-Meng Dai \\ College of Physics and Electronic Information Engineering, Wenzhou University, Wenzhou 325000, China \\ Correspondence should be addressed to Da-Meng Dai; daidameng1977@sina.cn
}

Received 15 January 2015; Revised 13 March 2015; Accepted 13 March 2015

Academic Editor: Hui Zhang

Copyright (C) 2015 Da-Meng Dai. This is an open access article distributed under the Creative Commons Attribution License, which permits unrestricted use, distribution, and reproduction in any medium, provided the original work is properly cited.

Due to the probability of the packet dropout in the networked control systems, a balanced reduced-order fault detection filter is proposed. In this paper, we first analyze the packet dropout effects in the networked control systems. Then, in order to obtain a robust fault detector for the packet dropout, we use the balanced structure to construct a reduced-order model for residual dynamics. Simulation results are provided to testify the proposed method.

\section{Introduction}

The background of the research is the increasing application of the wireless networks as medium in complex large-scale systems. In networked control systems (NCS), the control loops are close via communication networks. Hence, compared with traditional control systems, the NCS have many advantages, such as fewer system wiring, lower cost of maintenance, improvement of system flexibility, and application in the field of aerospace. So it has drawn more and more consideration in recent years. However, due to the insertion of the network, there appeared many challenging problems: the limited brand width of networked channel maybe cause impossibility of all information transmitted at the same time; due to the uncertainty, the information may be delayed or even dropped; the communication method of the networked system is the digital communication, so there may exist quantization error.

Since some packets have the probability of dropout in the channel, the detection of the faults may cause the false alarm for the NCS. Much work has been done to deal with this problem. In order to deal with the false alarm of the fault detection, the structure of standard model-based residual generator is suggested in [1], and then a residual evaluation scheme is developed to reduce the false alarm rate caused by the packet dropout. If the packets dropout is supposed to be finite, the dynamics of the observer error is modeled as a switched system. Also, the stability condition of observer is provided, and observer gain is obtained by solving LMI optimization problem [2]. Under the stochastic packet dropout in the network, the NCS are modeled as a Markovian jump linear system with four operation modes. Based on this model, the residual generator is developed and the fault detection is reformatted as a problem of $\mathrm{H}$ infinity filter [3]. When the packets dropout is described as discrete-time Markov jumping linear systems (MJLS), the stationary MJLS and the nonstationary MJLS are studied for fault detection problems [4]; then the problem of robust fault detection filter design and optimization is investigated with random delays in [5]. Under the condition of a short sampling period, the limited resource, and computational capacity, a reduced-order fault detection filter algorithm is proposed in [6]. To deal with the fault detection problems of nonlinear discrete-time NCS, the discrete observer based on sliding mode is designed to guarantee the condition of the specified sliding surface. Focusing on the networked control system with long time delays and data packet dropout, according to conditions of data arrival of the controller, the state observers of the system are designed to detect the faults in [7]. The fault detection filter is designed so that the overall fault detection dynamics is exponentially stable in the mean square 


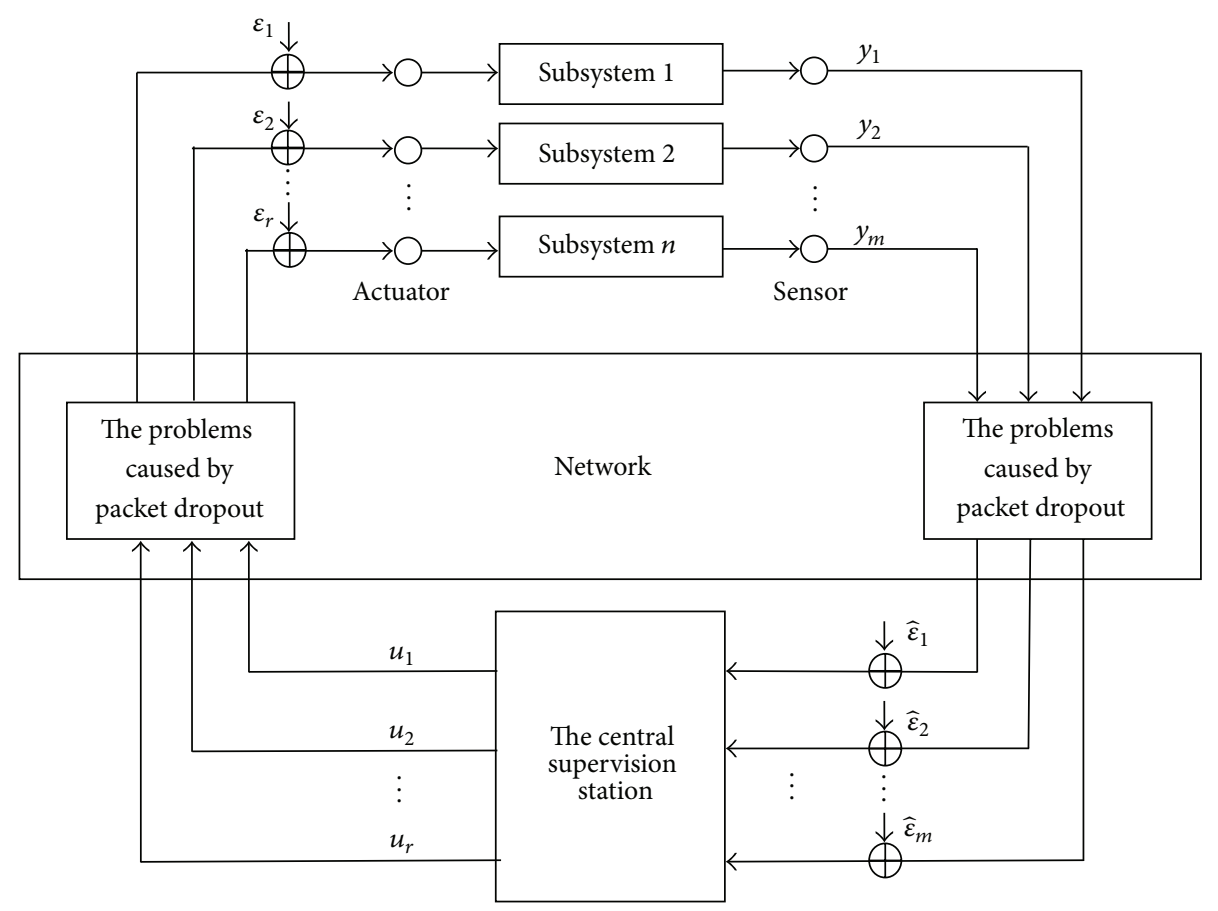

FIGURE 1: The structure of networked control systems.

with multiple communication delays and stochastic missing measurements.

$N$ th order linear time-invariant infinite impulse response system can be realized by infinite number methods, each has different sensitivity measurement. This property motivates us to seek a high performance realization in the fault detection for the NCS. Some results show that the characteristics of the parameterization play an important role in minimizing the output error $[8,9]$. Since the balanced realization, introduced by [10], has a good noise rejection characteristic, this property should lead the fault detection systems exhibiting well robust to the data packet dropout for the NCS.

For the fault detection system, the big Hankel singular values are more robust to the data packet dropout compared with the small ones; that is, the data packet dropout is easier to disturb the small Hankel singular values than the big ones. So we can improve the robust performance of the fault detection by the reduce-order model, that is, removing the small Hankel singular values. In this paper, in order to minimize the influence of the data packet dropout on the fault detection, we first analyze the packet dropout effects in the NCS. Then, based on the balanced structure, we construct a reduced-order fault detection filter and obtain the residual dynamics, which minimizes the ratio of maximumto-minimum eigenvalues of the Gramian matrices. It has low parameter sensitivity to the data packet dropout and the measurement noise and improves the robust to the false alarm. At last, the simulation results are provided to corroborate the analytical theory.

\section{Problem Description}

Figure 1 gives the structure of the NCS with the packet dropout. Assume that the dynamics of the subsystem to be monitored is a linear time-invariant system and can be described by the discrete model:

$$
\begin{gathered}
x(k+1)=\mathbf{A} x(k)+\mathbf{B}[u(k)+\varepsilon(k)]+\mathbf{E} f(k), \\
y(k)=\mathbf{C} x(k)+\mathbf{F} f(k),
\end{gathered}
$$

where $x, u$, and $y$ denote the system state, control input signal, and measurable output signal, respectively. A, B, C, E, and F are known constant matrices with appropriate dimensions. $f$ denotes the faults to be detected, and the measurement noise $\varepsilon$ is zero mean white noise for the input signal of the NCS.

The networks are between the sensor and the controller, and they have the same sample time and are simultaneous. Since the communication is hold in the network environment, we assume that there exists packets loss in the communication link and the signal where the central catch is given as follow:

$$
y^{a}(k)= \begin{cases}y(k)+\widehat{\varepsilon}(k), & \text { if } \gamma(k)=1, \\ \text { the last available measurement, } & \text { if } \gamma(k)=0,\end{cases}
$$

where $\gamma(k)$ describes the phenomenon of the packet dropout. $\gamma(k)=1$ means that the measurement at time point $k$ is correct, while $\gamma(k)=0$ means that this measurement is lost. When $\gamma(k)=0$, we use the last available measurement to 
generate the residual signal. The measurement noise $\widehat{\varepsilon}$ is zero mean white noise for the output signal of the NCS.

\section{Fault Detection Filter}

In this paper, we assume that the faults supervision stations and the central controller station are located together. Since the residual generator catches the input signal directly, in order to get the deviation, we must obtain the measurement output through the network. Hence, if there are some packets dropout which cause the network jam in the channel, some measurement output signal may be lost between the sensors and the supervision station. In order to obtain a robust residual generator and make the fault detection filter less sensitive to the packets dropout, we can remove the small Hankel singular values. So we design a reduced-order residual generator based on the balanced realization, and it is described as follow:

$$
\begin{gathered}
\widehat{x}(k+1)=\widehat{\mathbf{A}} \hat{x}(k)+\widehat{\mathbf{B}} u(k)+\mathbf{L}\left(y^{a}(k)-\widehat{y}(k)\right), \\
r(k)=\mathbf{W}\left(y^{a}(k)-\widehat{y}(k)\right), \\
\widehat{y}(k)=\widehat{\mathbf{C}} \hat{x}(k),
\end{gathered}
$$

where $\widehat{x}$ is the state of the fault detection filter, $\widehat{y}$ is the output signal of the filter, and $r$ is the residual signal. $\widehat{\mathbf{A}}, \widehat{\mathbf{B}}$, and $\widehat{\mathbf{C}}$ are the known matrices of the fault detection filter, which are deduced from the matrices $\mathbf{A}, \mathbf{B}$, and $\mathbf{C}$. $\mathbf{L}$ and $\mathbf{W}$ are vectors which ensure the stability and dynamics of the residual signal. The method used to select $\mathbf{L}$ and $\mathbf{W}$ in this paper is given by [11].

If we define that

$$
\chi(k)=y^{a}(k)-\widehat{y}(k)
$$

in ideal conditions, the result of $(4)$ is $\widehat{\varepsilon}(k)$. Thus, we suppose that the packet is dropout when the square of (4) is more than $2 \delta^{2}$; that is,

$$
\begin{aligned}
& \chi^{2}(k)>2 \delta^{2} \Longrightarrow \gamma(k)=0, \\
& \chi^{2}(k) \leq 2 \delta^{2} \Longrightarrow \gamma(k)=1,
\end{aligned}
$$

where $\delta^{2}$ is the variance of the white noise $\widehat{\varepsilon}$. In case of $\gamma(k)=$ 0 , we wish that the observation value is more close to the actual value, so we set the vector $\mathbf{L}$ to be zero; that is,

$$
\mathbf{L}=0, \quad \chi^{2}(k) \leq 2 \delta^{2} .
$$

We use the threshold selector to evaluate the residual generator (3). The decision is made based on the following logic:

$$
\begin{gathered}
J_{r}>J_{\mathrm{th}} \Longrightarrow \text { A fault is detected, } \\
J_{r} \leq J_{\mathrm{th}} \Longrightarrow \text { No fault, }
\end{gathered}
$$

where $J_{\text {th }}$ is a threshold, and it is determined by the method in [1]. The residual evaluation function $J_{r}$ is calculated by

$$
J_{r}=\left(\frac{1}{L} \sum_{i=0}^{L-1} r(k-i)\right)^{1 / 2}
$$

\section{Parameters of Residual Generator}

In this section, based on balanced reduced-order model, we aim to find the optimal system parameters for the fault detection filter. Because it can minimize the effects of the measurement noises $\varepsilon$ and $\widehat{\varepsilon}$. That is to say, we need a robust fault detection system. We consider that the subsystem is a discrete SISO system. Thus, based on (1), we have

$$
\begin{aligned}
G(z) & =\mathbf{C}(z \mathbf{I}-\mathbf{A})^{-1} \mathbf{B}=\frac{q_{N}(z)}{p_{N}(z)} \\
& =\frac{b_{0}+b_{1} z^{-1}+\cdots+b_{N} z^{-N}}{1+a_{1} z^{-1}+\cdots a_{N} z^{-N}},
\end{aligned}
$$

where the coefficients $b_{0}, b_{1}, \ldots, b_{N}$ and $a_{1}, a_{2}, \ldots, a_{N}$ denote the corresponding system input and output variables. In order to obtain the balanced reduced-order fault detection model of the NCS, using the result in $[12,13]$, we firstly construct the system $(\widetilde{\mathbf{A}}, \widetilde{\mathbf{B}}, \widetilde{\mathbf{C}})$ to be

$$
\begin{gathered}
\widetilde{\mathbf{A}}=\left(\begin{array}{cccc}
-a_{1} & \cdots & -a_{N-1} & -a_{N} \\
1 & \cdots & 0 & 0 \\
\vdots & \ddots & \vdots & \vdots \\
0 & \cdots & 1 & 0
\end{array}\right), \\
\widetilde{\mathbf{B}}=\left(\begin{array}{c}
1 \\
0 \\
\vdots \\
0
\end{array}\right), \quad \widetilde{\mathbf{C}}=\left(\begin{array}{c}
b_{1}-b_{0} a_{1} \\
b_{2}-b_{0} a_{2} \\
\vdots \\
b_{N}-b_{0} a_{N}
\end{array}\right) .
\end{gathered}
$$

Given the polynomial as

$$
\widetilde{p}_{N}(z)=z^{N} p_{N}(z)=z^{N}+a_{1} z^{N-1}+\cdots+a_{N}
$$

and the shift matrix $\mathbf{Z}=R^{N \times N}$ as

$$
\mathbf{Z}=\left(\begin{array}{cccc}
0 & 1 & \cdots & 0 \\
\vdots & \vdots & \ddots & \vdots \\
0 & 0 & \cdots & 1 \\
0 & 0 & \cdots & 0
\end{array}\right),
$$

then we can get the Schur-Cohn matrix:

$$
\mathbf{H}=\widetilde{p}_{N}(\mathbf{Z})^{T} \tilde{p}_{N}(\mathbf{Z})-p_{N}(\mathbf{Z})^{T} p_{N}(\mathbf{Z}) .
$$

Define the matrix $\mathbf{M}=\left\{m_{i, j}\right\}_{N \times N}$ as

$$
m_{i, j}=\sum_{\tau \geq 1} a_{i-\tau} c_{j+\tau-1}-\sum_{\tau \geq 1} c_{i-\tau} a_{j+\tau-1}
$$

where $a_{0}=1, c_{0}=0$, and $c_{i}=b_{i}-b_{0} a_{i},(1 \leq i \leq N)$.

Then we define

$$
\mathbf{W}_{c o}=\mathbf{H}^{-1} \mathbf{M}=\mathbf{V} \Lambda \mathbf{V}^{-1},
$$


where $\mathbf{\Lambda}$ and $\mathbf{V}$ are the eigenvalue and eigenvector matrices of $\mathbf{W}_{c o}$, respectively. The matrix $\mathbf{Q}$ is defined as

$$
\mathbf{Q}=\mathbf{V}^{\mathrm{T}} \mathbf{M V}
$$

in which the diagonal elements are $q_{1}, q_{2}, \ldots, q_{N}$. As a result, we define the matrix $\mathbf{F}$ as

$$
\mathbf{F}=\operatorname{diag}\left(\left|q_{1}\right|^{-1 / 2},\left|q_{2}\right|^{-1 / 2}, \ldots,\left|q_{N}\right|^{-1 / 2}\right)
$$

From (15) and (17), we can obtain the transformation $\mathbf{T}_{\text {bal }}$ from controllable canonical to a balanced form as $[12,13]$

$$
\mathrm{T}_{\text {bal }}=\mathrm{VF} \text {. }
$$

Now, the balanced realization system $\left(\mathbf{A}_{\text {bal }}, \mathbf{B}_{\text {bal }}, \mathbf{C}_{\text {bal }}\right)$ can be calculated as follow:

$$
\begin{gathered}
\mathbf{A}_{\text {bal }}=\mathbf{T}_{\text {bal }}^{-1} \widetilde{\mathbf{A}} \mathbf{T}_{\text {bal }}, \\
\mathbf{B}_{\text {bal }}=\mathbf{T}_{\text {bal }}^{-1} \widetilde{\mathbf{B}}, \\
\mathbf{C}_{\text {bal }}=\widetilde{\mathbf{C}} \mathbf{T}_{\text {bal }} .
\end{gathered}
$$

The controllability Gramian $\mathbf{W}_{c}$ and the observability Gramian $\mathbf{W}_{o}$ of the system $\left(\mathbf{A}_{\text {bal }}, \mathbf{B}_{\text {bal }}, \mathbf{C}_{\text {bal }}\right)$ are equal to each other. Consider

$$
\mathbf{W}_{c}=\mathbf{W}_{o}=\operatorname{diag}\left(\sigma_{1}, \sigma_{2}, \ldots, \sigma_{N}\right),
$$

where $\sigma_{1}>\sigma_{2}>\cdots>\sigma_{N-1}>\sigma_{N}>0$. In order to obtain a balanced reduced-order system, we partition the system $\left(\mathbf{A}_{\text {bal }}, \mathbf{B}_{\text {bal }}, \mathbf{C}_{\text {bal }}\right)$ as

$$
G_{\text {bal }} \sim\left[\begin{array}{cc|c}
\mathbf{A}_{(N-M) \times(N-M)}^{11} & \mathbf{A}_{(N-M) \times M}^{12} & \mathbf{B}_{(N-M) \times 1}^{1} \\
\mathbf{A}_{M \times(N-M)}^{21} & \mathbf{A}_{M \times M}^{22} & \mathbf{B}_{M \times 1}^{2} \\
\hline \mathbf{C}_{1 \times(N-M)}^{1} & \mathbf{C}_{1 \times M}^{2} & \mathbf{0}
\end{array}\right] .
$$

From (21), we obtain the reduced-order model for the networked control systems $\left(\mathbf{A}_{(N-M) \times(N-M)}^{11}, \mathbf{B}_{(N-M) \times 1}^{1}\right.$, $\left.\mathbf{C}_{1 \times(N-M)}^{1}\right)$, which is still a balanced realization. Using this result in (3), that is, setting the system $(\widehat{\mathbf{A}}, \widehat{\mathbf{B}}, \widehat{\mathbf{C}})$ to be $\left(\mathbf{A}_{(N-M) \times(N-M)}^{11}, \mathbf{B}_{(N-M) \times 1}^{1}, \mathbf{C}_{1 \times(N-M)}^{1}\right)$, the balanced reducedorder fault detection filter for the NCS is obtained, which minimizes the ratio of maximum-to-minimum eigenvalues of the Gramian matrices and has a good packets dropout rejection.

\section{Simulation Results}

In this section, we analyze the proposed algorithm in MATLAB. The first case of the NCS is the fourth-order system, and the matrices are given as

$$
\begin{aligned}
& \mathbf{A}=\left[\begin{array}{cccc}
-0.2571 & 0.2350 & 0.5290 & 0.1497 \\
-0.6290 & -0.2154 & 0.0990 & -0.2897 \\
0.1059 & -0.2623 & -0.2553 & -0.5475 \\
0.1690 & 0.5330 & 0.0760 & -0.2820
\end{array}\right] \text {, } \\
& \mathbf{B}=\left[\begin{array}{c}
0.1258 \\
0.2175 \\
-0.5990 \\
-0.2430
\end{array}\right] \\
& \mathrm{C}=\left[\begin{array}{llll}
0.1190 & -0.2210 & 0.2307 & -0.6274
\end{array}\right] \text {, } \\
& \mathbf{E}=\left[\begin{array}{c}
0.5258 \\
0.6175 \\
-0.59909 \\
-0.6430
\end{array}\right], \quad \mathbf{F}=0.3984
\end{aligned}
$$

Since the dropout of the packets is random, we use twostate Markov jump system to describe the stochastic process [1]. And the parameter $\gamma(k) \in\{0,1\}$ denotes the packet dropout or not, so the probability of the state transition can be described as

$$
\begin{aligned}
& \lambda_{i j}=\operatorname{Prob}\{\gamma(k+1)=j \mid \gamma(k)=i\}, \\
& \sum_{j=0}^{1} \lambda_{i j}=1, \quad \forall i, j \in\{0,1\}, \lambda_{i j} \geq 0 .
\end{aligned}
$$

And the state transfer matrix of the Markov chain is given as

$$
\left[\lambda_{i j}\right]_{i, j=0,1}=\left[\begin{array}{ll}
0.5 & 0.5 \\
0.3 & 0.7
\end{array}\right]
$$

The measurement noises $\varepsilon(k)$ and $\widehat{\varepsilon}(k)$ are the Gaussian white noise with the variance 0.2 . The fault signal is described as

$$
f(k)= \begin{cases}1+0.5 \cos (4 \pi k), & \text { for } k=2000,2001, \ldots, 5000 \\ 0, & \text { else. }\end{cases}
$$




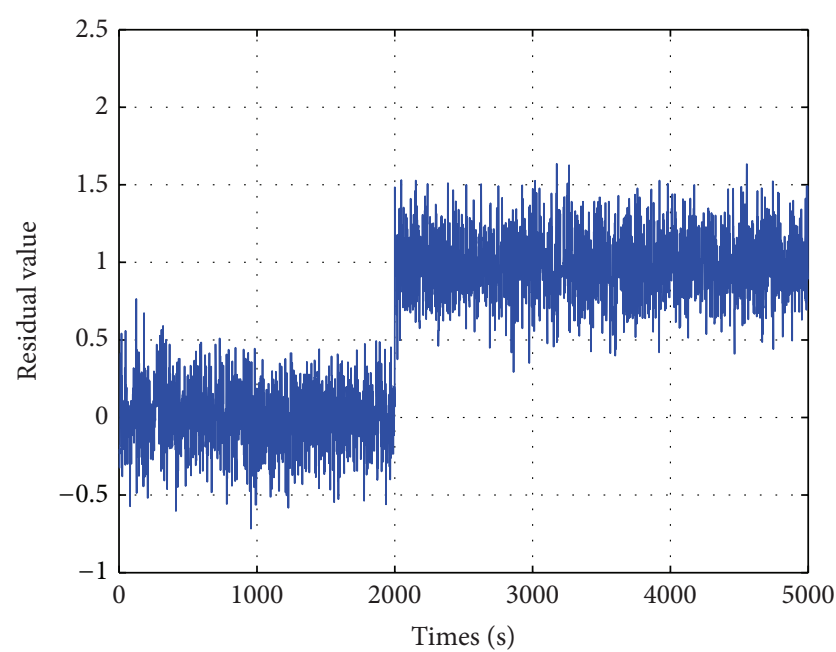

FIGURE 2: Residual signal of the initial system given by (22) using (25).

By using (21), we can get the following system, in which the system matrices are

$$
\begin{gathered}
\widehat{\mathbf{A}}=\left[\begin{array}{ccc}
-0.2571 & 0.5290 & 0.1497 \\
0.1059 & -0.2553 & -0.5475 \\
0.1690 & 0.0760 & -0.2820
\end{array}\right], \\
\widehat{\mathbf{B}}=\left[\begin{array}{c}
0.1258 \\
-0.5990 \\
-0.2430
\end{array}\right], \\
\widehat{\mathbf{C}}=\left[\begin{array}{lll}
0.1190 & 0.2307 & -0.6274
\end{array}\right] .
\end{gathered}
$$

The residual generators of the initial system given by (22) and the balanced reduced-order system given by (26) are shown in Figures 2 and 3, respectively. From Figures 2 and 3, we can conclude that the residual generator of the balanced reduced-order system gives the accurate fault alarm and has a good packets dropout and measurement noise rejection.

When the fault signal $f$ is given as

$$
f(k)= \begin{cases}0.5 \sin k, & \text { for } k=1500,1501, \ldots, 5000 \\ 0, & \text { else }\end{cases}
$$

the measurement noise is

$$
\widetilde{\varepsilon}(k)=2 e^{(-0.0006 k)} n(k),
$$

where $n(k)$ and $\varepsilon(k)$ are the Gaussian white noise with the variances 0.01 and 0.2 , respectively. Figures 4 and 5 give the residual signal of the initial system and the balanced reducedorder system. We find that the balanced reduced-order residual generator show a good robust to the measurement noise and the packet dropout, and it also gives the accurate faults alarm.

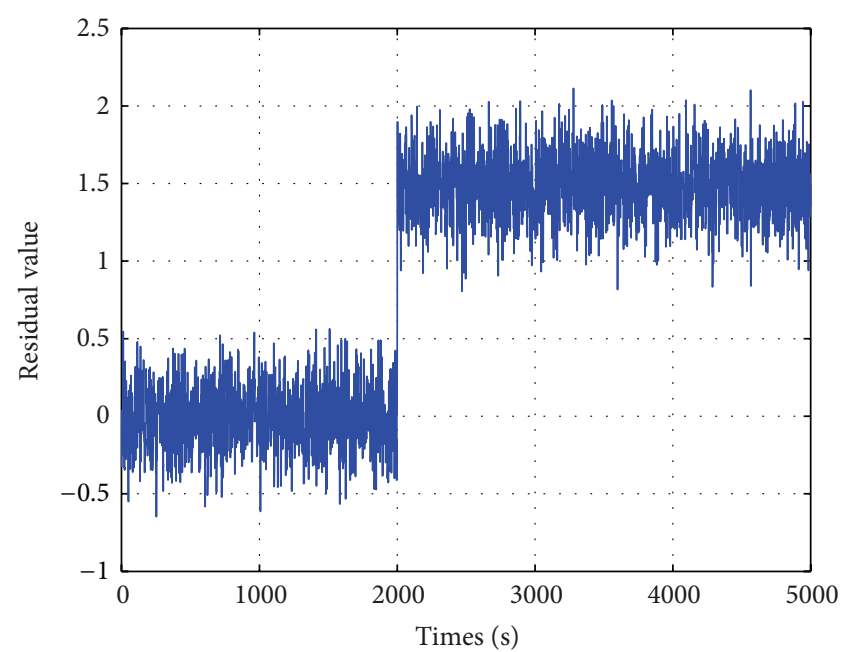

FIGURE 3: Residual signal of the reduced-order system given by (26) using (25).

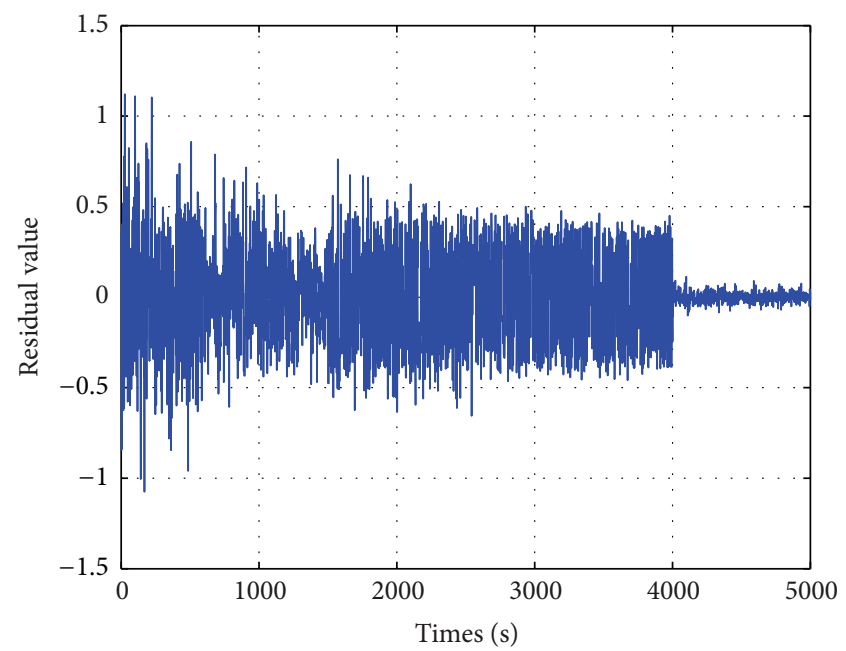

FIGURE 4: Residual signal of the initial system given by (22) using (27).

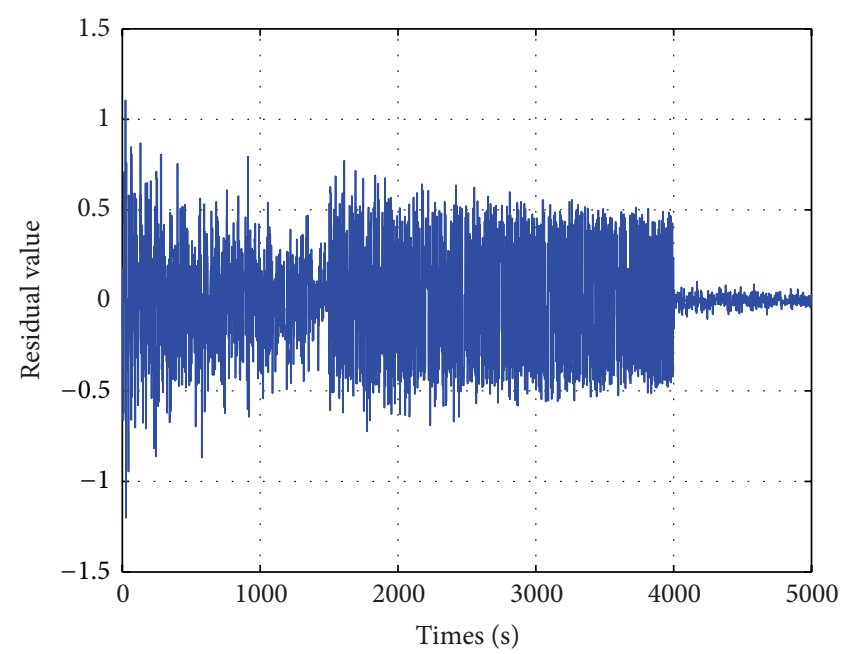

FIGURE 5: Residual signal of the reduced-order system given by (26) using (27). 
The second case system is the fifth-order system, and the matrices of the NCS are given as follows:

$$
\begin{aligned}
& \mathbf{A}=\left[\begin{array}{ccccc}
-0.6571 & 0.9250 & 0.8290 & 0.0497 & 0.8535 \\
-0.0290 & -0.5154 & 0.0990 & 0.0897 & 0.0636 \\
0.1059 & -0.9623 & 0.2553 & -0.3750 & 0.2456 \\
0.1690 & 0.2330 & 0.1760 & -0.0820 & 0.7493 \\
0.0345 & -0.5433 & 0.6046 & 0.4237 & 0.3986
\end{array}\right] \\
& \mathbf{B}=\left[\begin{array}{c}
0.1258 \\
0.2175 \\
-0.1990 \\
-0.2430 \\
0.6673
\end{array}\right] \\
& \mathrm{C}=\left[\begin{array}{lllll}
0.1190 & -0.2210 & 0.2307 & -0.6274 & 0.6490
\end{array}\right], \\
& \mathbf{E}=\left[\begin{array}{c}
0.8258 \\
0.6175 \\
-0.7990 \\
-0.5430 \\
0.8053
\end{array}\right], \quad \mathbf{F}=0.1984
\end{aligned}
$$

Based on (21), we can obtain the fourth-order system as follows:

$$
\begin{aligned}
& \widehat{\mathbf{A}}=\left[\begin{array}{cccc}
-0.6571 & 0.9250 & 0.0497 & 0.8535 \\
-0.0290 & -0.5154 & 0.0897 & 0.0636 \\
0.1690 & 0.2330 & -0.0820 & 0.7493 \\
0.0345 & -0.5433 & 0.4237 & 0.3986
\end{array}\right], \\
& \widehat{\mathbf{B}}=\left[\begin{array}{c}
0.1258 \\
0.2175 \\
-0.2430 \\
0.6673
\end{array}\right] \\
& \widehat{\mathbf{C}}=\left[\begin{array}{llll}
0.1190 & -0.2210 & -0.6274 & 0.6490
\end{array}\right], \\
& \widehat{\mathbf{E}}=\left[\begin{array}{c}
0.8258 \\
0.6175 \\
-0.5430 \\
0.8053
\end{array}\right], \quad \widehat{\mathbf{F}}=0.1984
\end{aligned}
$$

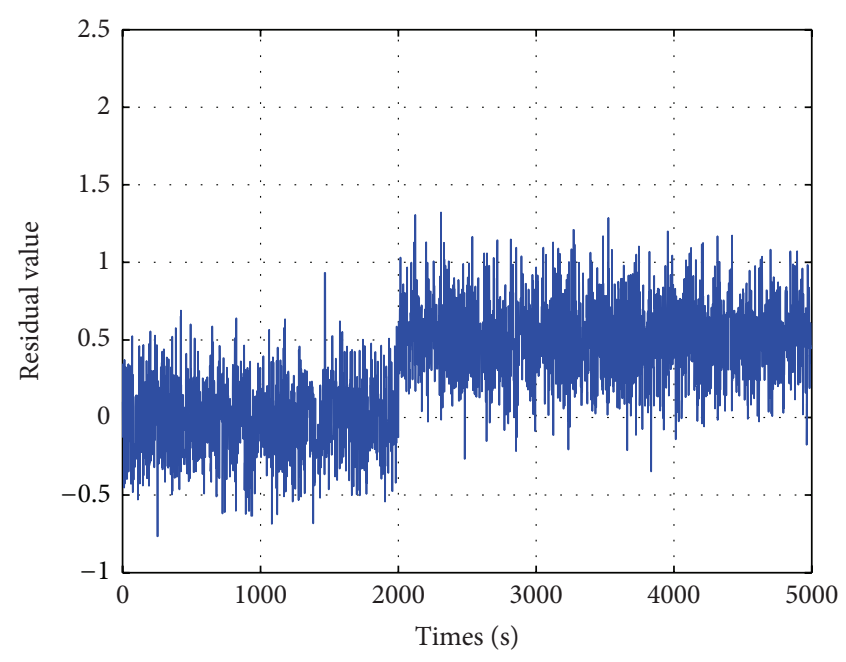

FIgURE 6: Residual signal of the initial system given by (29) using (25).

The same as (30), using (21), the third-order system can be obtained as

$$
\begin{aligned}
& \widehat{\mathbf{A}}_{2}=\left[\begin{array}{ccc}
-0.6571 & 0.9250 & 0.8535 \\
-0.0290 & -0.5154 & 0.0636 \\
0.0345 & -0.5433 & 0.3986
\end{array}\right] \text {, } \\
& \widehat{\mathbf{B}}_{2}=\left[\begin{array}{l}
0.1258 \\
0.2175 \\
0.6673
\end{array}\right] \\
& \widehat{\mathbf{C}}_{2}=\left[\begin{array}{lll}
0.1190 & -0.2210 & 0.6490
\end{array}\right], \\
& \widehat{\mathbf{E}}_{2}=\left[\begin{array}{l}
0.8258 \\
0.6175 \\
0.8053
\end{array}\right], \quad \widehat{\mathbf{F}}_{2}=0.1984 \text {. }
\end{aligned}
$$

When the measurement noises $\varepsilon(k)$ and $\widehat{\varepsilon}(k)$ are the Gaussian white noise with the variance 0.2 , the fault signal is described as (25). The residual signal $r(k)$ of the initial system given by (29) is shown in Figure 6, and the residual signal of the balanced reduced-order system given by (30) and (31) are shown in Figures 7 and 8, respectively. From Figures 6, 7, and 8 , we find that the residual generator of the reduced-order system given by (30) and (31) shows more accurate alarm because the small Hankel singular values are removed and the robust of the systems (30) and (31) are improved. When the fault signal is described as (27) and the measurement noise is given as (28). Figures 9, 10, and 11 give the simulation results of the residual signal. From these three figures, we find that the residual signal of the initial system confuse with the noise at interval [1000,2000], while the balanced reduced-order systems given by (30) and (31) can easily distinguish the fault signal since they remove the small Hankel singular values and have the better measurement noise and packet dropout rejection. 


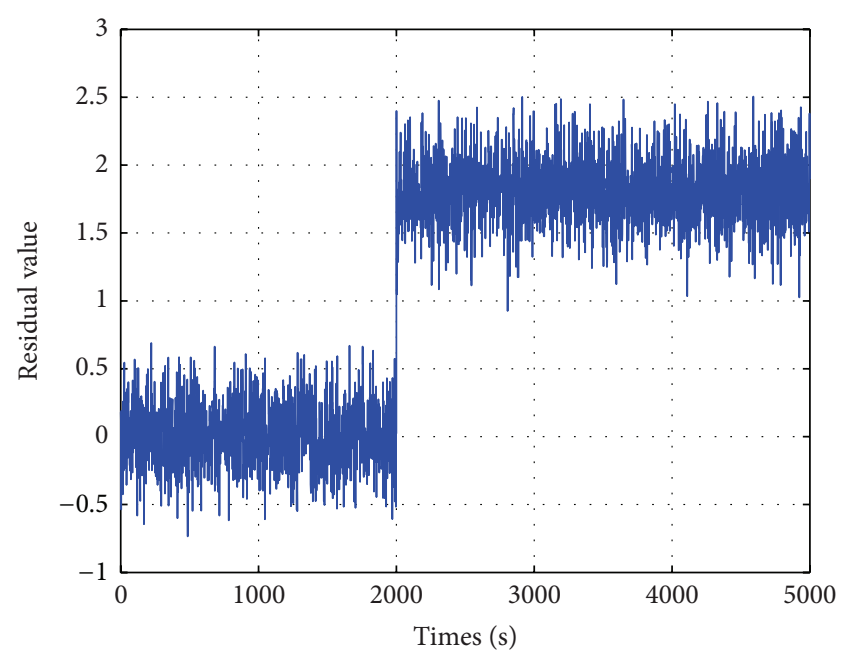

FIGURE 7: Residual signal of the reduced-order system given by (30) using (25).

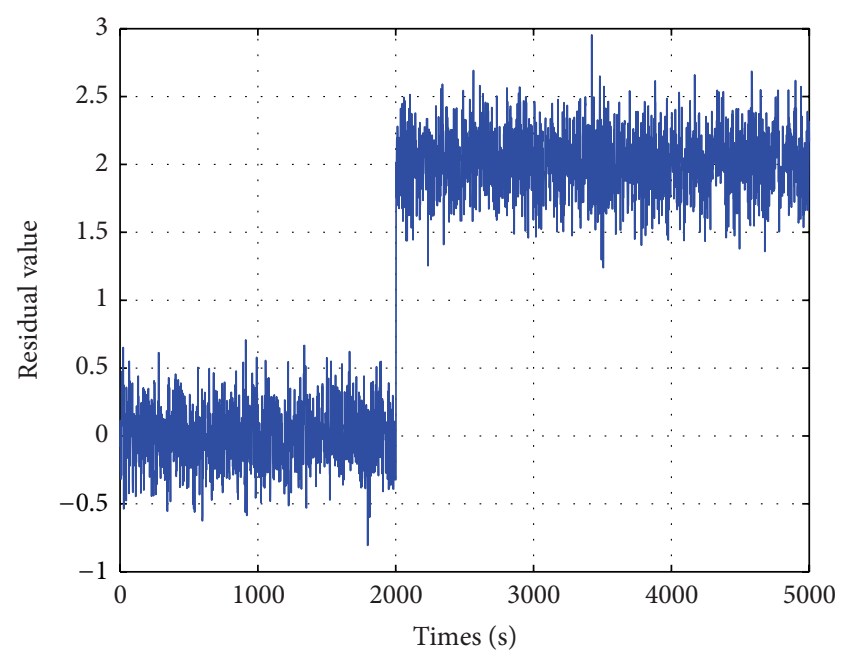

FIGURE 8: Residual signal of the reduced-order system given by (31) using (25).

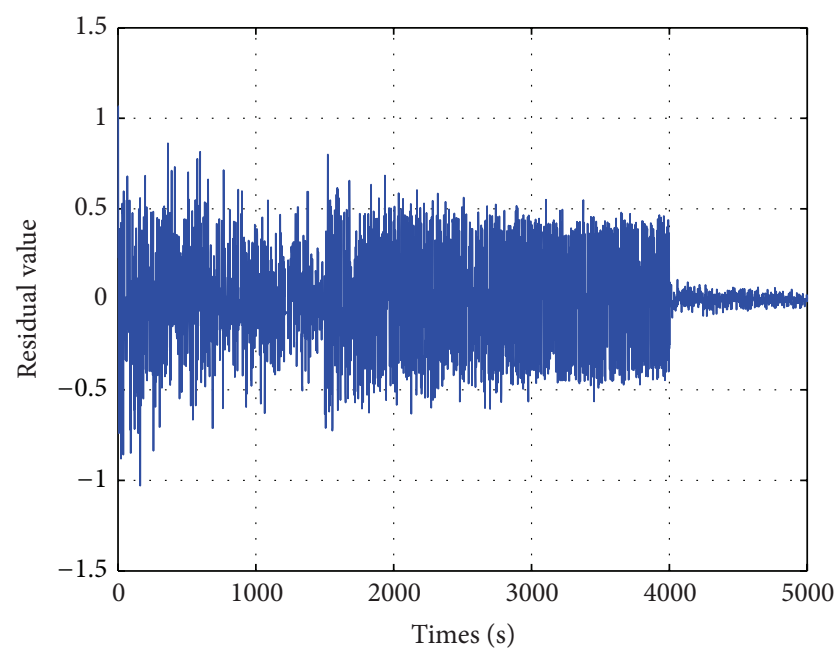

FIGURE 9: Residual signal of the initial system given by (29) using (27).

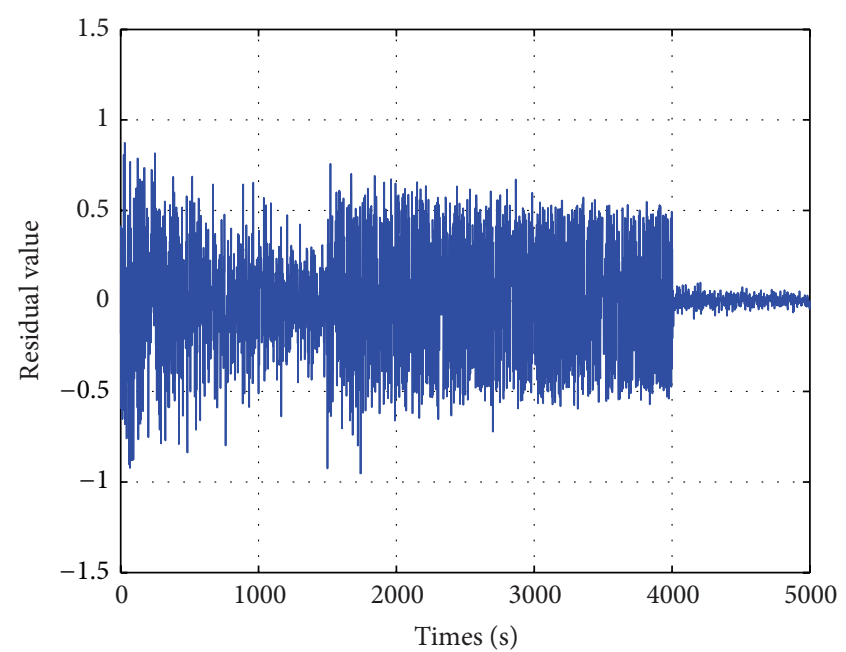

FIGURE 10: Residual signal of the reduced-order system given by (30) using (27).

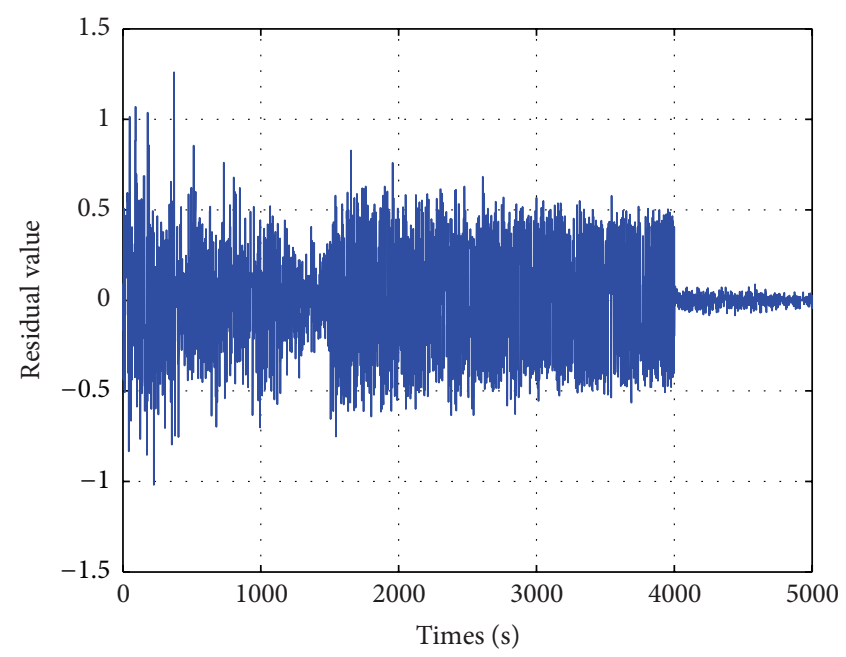

FIGURE 11: Residual signal of the reduced-order system given by (31) using (27).

\section{Conclusions}

The packets dropout is inevitable in the NCS; we analyze the effects of this phenomenon for the fault detection systems. Since the balanced reduced-order model minimizes the ratio of maximum-to-minimum eigenvalues of the Gramian matrices and has low parameter sensitivity to the data packet dropout and the measurement noise, we propose a new structure of the fault detection for the NCS based on the balanced realization, which lead this structure to have a good packets dropout and measurement noise rejection. Simulation results show that the proposed method has a good performance for the NCS. For future work, the intelligent design could be further included in the scheme to deal with the case of system learning. For future work, the intelligent design [14-16] could be further 
included in the scheme to deal with the case of system learning.

\section{Conflict of Interests}

The author declares that there is no conflict of interests.

\section{Acknowledgments}

This work was supported in part by the Key Project of Natural Science Foundation of Zhejiang Province under Grant no. LZ14F010002 and in part by the Public Welfare Project of Zhejiang Province under Grants nos. 2012C21048 and 2012 C23040.

\section{References}

[1] P. Zhang, S. X. Ding, P. M. Frank, and M. Sader, "Fault detection of networked control systems with missing measurements," in Proceedings of the 5th Asian Control Conference, pp. 1258-1263, Melbourne, Australia, July 2004.

[2] Z. Li, D. Sun, J. J. Zhen, and Y. T. Shi, "Fault detection for networked control system with data packet dropout," in Proceedings of the IEEE International Conference on Automation and Logistics (ICAL '09), pp. 613-616, IEEE, Shenyang, China, August 2009.

[3] H. Huang, D. X. Xie, X. D. Han, D. F. Zhang, and Z. Q. Wang, "Fault detection for networked control system with random packet dropout," Control Theory and Applications, vol. 28, no. 1, pp. 79-86, 2011.

[4] W. Li, F. Jiang, Z. Wang, G. Zhou, and Z. Zhu, "Fault detection of Markov jumping linear systems," Mathematical Problems in Engineering, vol. 2012, Article ID 141867, 27 pages, 2012.

[5] W. L. Jiang, C. Y. Dong, E. Z. Niu, and Q. Wang, "Observerbased robust fault detection filter design and optimization for networked control systems," Mathematical Problems in Engineering. In press.

[6] Q. Wang, M.-M. Wang, and C.-Y. Dong, "Design of a kind of reduced-order fault detection filtering algorithm and its application in space vehicles," Journal of Astronautics, vol. 34, no. 1, pp. 61-68, 2013.

[7] X. Li, X. B. Wu, Z. L. Xu, and C. Huang, "Fault detection observer design for networked control system with long timedelays and data packet dropout," Journal of Systems Engineering and Electronics, vol. 21, no. 5, pp. 877-882, 2010.

[8] P. A. Regalia, Adaptive IIR Filtering in Signal Processing and Control, Marcel Dekker, New York, NY, USA, 1995.

[9] Y. F. Zhi, X. Zheng, and R. Li, "On the convergence behavior of affine projection algorithm with direction error," Asian Journal of Control, vol. 16, no. 2, pp. 530-538, 2014.

[10] B. C. Moore, "Principal component analysis in linear systems: controllability, observability, and model reduction," IEEE Transactions on Automatic Control, vol. 26, no. 1, pp. 17-32, 1981.

[11] P. M. Frank and X. Ding, "Frequency domain approach to optimally robust residual generation and evaluation for modelbased fault diagnosis," Automatica, vol. 30, no. 5, pp. 789-804, 1994.

[12] K. Hochan, C. Chongho, O. Sangrok, and K. Kwangbae, "Minimal balanced realization of SISO systems using closedform Gramians," in Proceedings of the Proceedings of the 1995
American Control Conference, vol. 3, pp. 1837-1841, IEEE, Seattle, Wash, USA, June 1995.

[13] Y. F. Zhi, P. G. Fan, and J. Zhang, "Adaptive IIR filtering based on balanced-realization," Journal of Systems Engineering and Electronics, vol. 24, no. 1, pp. 19-25, 2013.

[14] B. Xu, C. G. Yang, and Z. K. Shi, "Reinforcement learning output feedback NN control using deterministic learning technique," IEEE Transactions on Neural Networks and Learning Systems, vol. 25, no. 3, pp. 635-641, 2014.

[15] B. Xu, Z. K. Shi, C. G. Yang, and F. C. Sun, "Composite neural dynamic surface control of a class of uncertain nonlinear systems in strict-feedback form," IEEE Transactions on Cybernetics, vol. 44, no. 12, pp. 2626-2634, 2014.

[16] B. Xu, Z. K. Shi, and C. G. Yang, "Composite fuzzy control of a class of uncertain nonlinear systems with disturbance observer," Nonlinear Dynamics, 2015. 


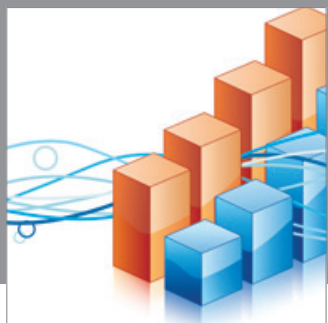

Advances in

Operations Research

mansans

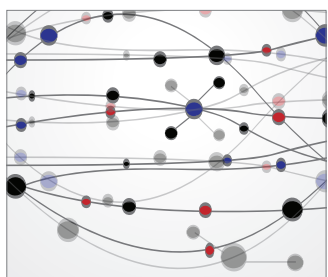

The Scientific World Journal
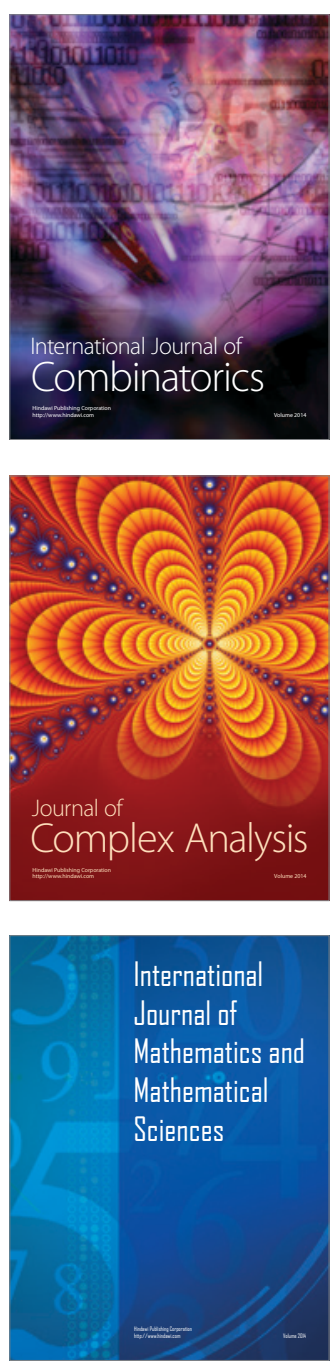
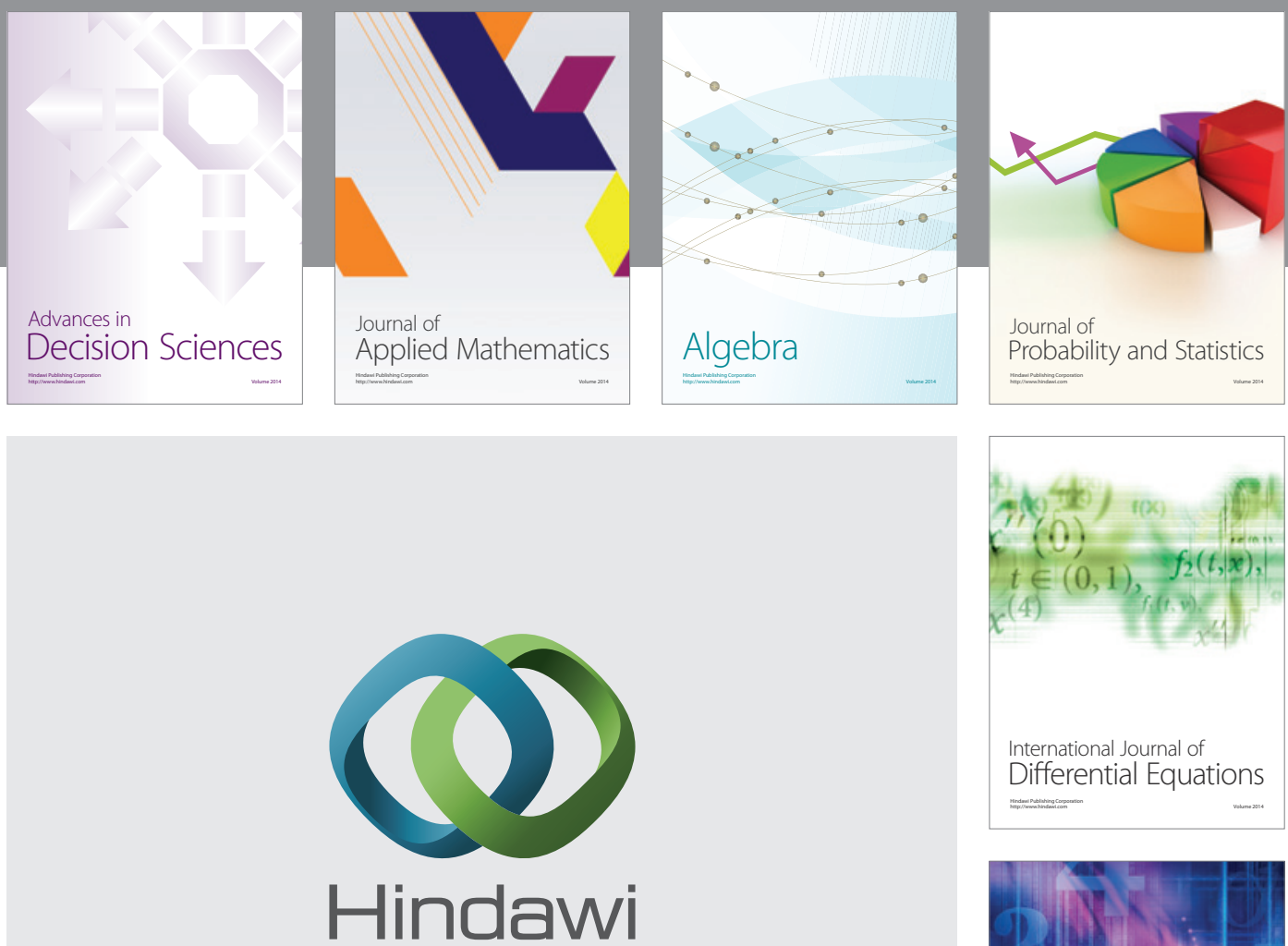

Submit your manuscripts at http://www.hindawi.com
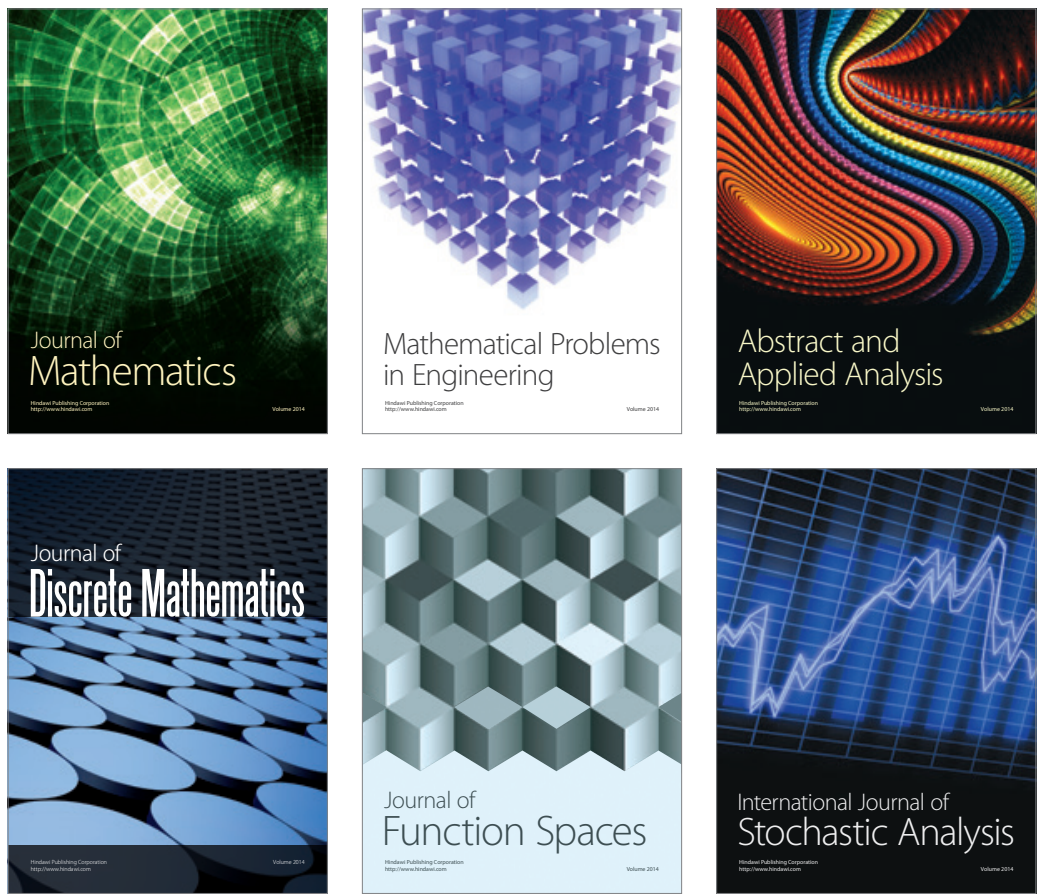

Journal of

Function Spaces

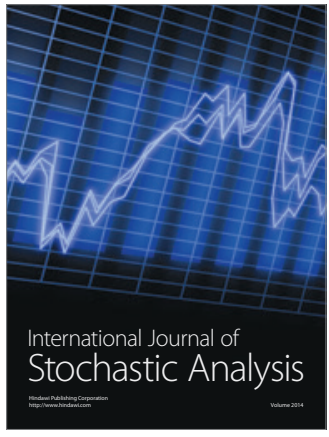

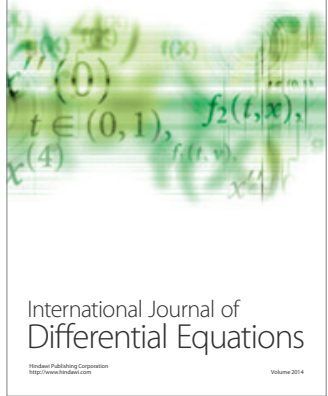
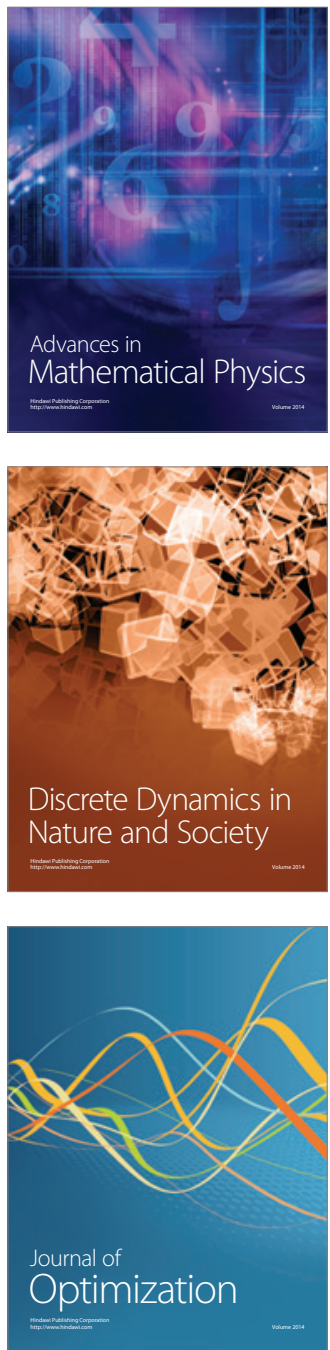